\title{
The perils of antiepileptic toxicity
}

\section{Case study}

A 63-year-old high-level-care nursing home resident was brought to hospital with reported 'seizures' at the facility. He had been increasingly lethargic and nonverbal for four days. The man had a history of epilepsy related to traumatic brain injury, alcohol-associated pancreatitis and Wernicke-Korsakoff's syndrome. He was cachectic. His regular treatment included valproate $1.5 \mathrm{~g}$ twice daily and levetiracetam $1.5 \mathrm{~g}$ twice daily.

Non-convulsive status epilepticus was suspected after a failure to regain consciousness. This was the provisional diagnosis as an EEG eight months previously was reported as showing non-convulsive status epilepticus.

The patient was given intravenous doses of valproate (800 mg), levetiracetam ( $1.5 \mathrm{~g}$ ) and phenytoin $(1.2 \mathrm{~g})$ in the emergency department. Before administration of these loading doses his total valproate concentration was $62 \mathrm{mg} / \mathrm{L}$, which is within the therapeutic range.

Over the subsequent two weeks, the patient remained unresponsive with no observed seizures. As this was assumed to be because of non-convulsive status epilepticus, twice daily phenytoin (200 mg) and lacosamide (100 $\mathrm{mg}$ ) had been added to his treatment. EEGs showed moderate-severe diffuse cortical dysfunction, but no electrographic seizure activity. Repeat total plasma concentrations on day 16 showed phenytoin $16 \mathrm{mg} / \mathrm{L}$ and valproate $21 \mathrm{mg} / \mathrm{L}$. In a review of the case, hypoalbuminaemia-adjusted total drug concentrations were calculated because phenytoin and valproate are both highly proteinbound drugs with the potential for concentrationdependent toxicity. His albumin on admission had been $23 \mathrm{~g} / \mathrm{L}$. The Figure shows the measured and hypoalbuminaemia-adjusted total drug concentrations. ${ }^{1,2}$ Given the correlation of the patient's clinical state and these results, it was realised that the patient had anticonvulsant toxicity.

Following cessation of phenytoin and reduction of the valproate dose, the patient recovered to his usual state. He was able to return to the residential care facility.

\section{Comment}

This case highlights concerns regarding recognising antiepileptic toxicity and interpreting drug concentrations in patients with hypoalbuminaemia, hepatic dysfunction and drug interactions.
The goal of antiepileptic therapy is to maximise seizure control and minimise adverse effects. As treatment aims to prevent seizures, it is difficult to clinically assess the lowest effective maintenance dose for long-term seizure control or recognise signs of toxicity. ${ }^{3}$ It is possible that the cause of the patient's increasing lethargy before admission was emerging anticonvulsant toxicity.

Phenytoin and valproate are both highly protein-bound drugs ( $>90 \%$ ) with non-linear pharmacokinetics, saturable protein binding and complex drug interactions. ${ }^{2}$ The free unbound component of the drugs is responsible for their antiepileptic activity and neurological and systemic toxicity. The risk of toxicity increases with the severity of hypoalbuminaemia. With both antiepileptics, toxicity may present as central nervous system depression, cerebellar dysfunction, seizures, hepatotoxicity and bone marrow abnormalities (Table) ${ }^{3,4}$

Valproate inhibits phenytoin metabolism and causes displacement of phenytoin from albumin, so it increases free phenytoin concentrations. Phenytoin induces valproate metabolism. This explains the decrease in valproate concentration once phenytoin was added and the significant increase once phenytoin was ceased (Fig.). ${ }^{3}$

\section{Monitoring}

Therapeutic drug monitoring is the measurement of drug concentrations with appropriate interpretation to influence prescribing. ${ }^{3}$ While commonly used to assess adherence, it is also useful when suspecting toxicity, establishing optimal drug dosing when starting therapy, adjusting doses or when using drugs that may interact. Monitoring is especially important for individuals with altered pharmacokinetics including patients with hypoalbuminaemia, underlying organ dysfunction and those at extremes of age or during pregnancy. ${ }^{3}$

Plasma concentrations of antiepileptic drugs correlate with adverse effects and for hypoalbuminaemic patients, free drug concentrations correlate better than total concentrations. Free concentrations more accurately reflect the amount of active drug within the brain. ${ }^{3}$ The upper limit of the reference range reflects the concentration above which there is an increased risk of toxicity (Table). ${ }^{4}$

\section{Arushi Madan \\ Clinical pharmacology advanced trainee \\ Tracy Risetto \\ Medicines information pharmacist, Queensland Medicines Advice and Information Service}

\section{Helen Trenerry}

Director of Queensland Medicines Advice and Information Service

\section{Peter Donovan}

Director of Clinical

Pharmacology

Royal Brisbane and

Women's Hospital, Brisbane

\section{Keywords}

anticonvulsive drugs, phenytoin, valproate

Aust Prescr 2020;43:61-3 https://doi.org/10.18773/ austprescr.2020.012 


\section{Fig. Antiepileptic toxicity in a patient with hypoalbuminaemia}
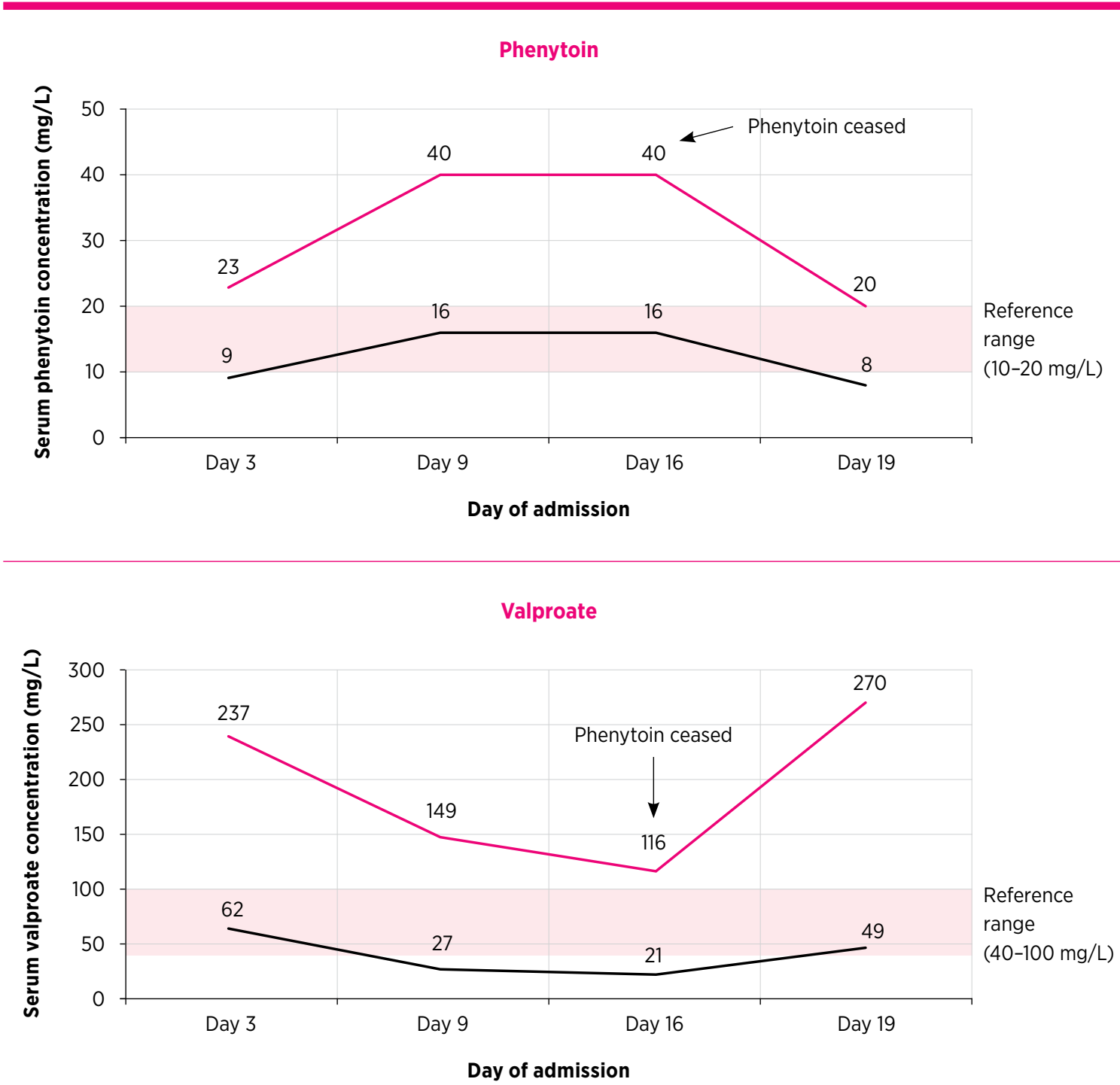

\section{Conclusion}

While therapeutic drug monitoring for phenytoin and valproate is widely available, most Australian laboratories measure total rather than free concentrations as it is faster and less expensive. However, the pharmacokinetic implications of hypoalbuminaemia may result in toxicity despite anticonvulsant concentrations within the therapeutic range. The Sheiner-Tozer equation for phenytoin and the Hermida-Tutor equation for valproate may be used to adjust the total concentrations for hypoalbuminaemia. However, these adjustments may still underestimate total and free drug concentrations in critically ill patients or those with multiorgan dysfunction.1,2 Our recommendation is to measure free drug concentrations in hypoalbuminaemic patients to monitor for potential drug-related toxicity. It is also important to remember that adverse drug reactions can present as new clinical problems.

Conflict of interest: none declared 


\section{Table Correlation of total plasma concentration and clinical features of toxicity for phenytoin and valproate}

\section{Clinical features of toxicity}

\section{Phenytoin}

\begin{tabular}{ll}
\hline $10-20 \mathrm{mg} / \mathrm{L}$ & Therapeutic range \\
$>20 \mathrm{mg} / \mathrm{L}$ & Nystagmus and ataxia \\
$>30 \mathrm{mg} / \mathrm{L}$ & Severe ataxia, dysarthria, hyperreflexia, drowsiness, nausea and vomiting \\
$>50 \mathrm{mg} / \mathrm{L}$ & $\begin{array}{l}\text { Extreme lethargy, coma, paradoxical seizures } \\
\text { Cardiac conduction abnormalities with intravenous administration only }\end{array}$
\end{tabular}

\section{Valproate}

\begin{tabular}{ll}
\hline $40-100 \mathrm{mg} / \mathrm{L}$ & Therapeutic range \\
$>100 \mathrm{mg} / \mathrm{L}$ & $\begin{array}{l}\text { Mild drowsiness and ataxia } \\
\text { Variable central nervous system depression }\end{array}$ \\
$>500 \mathrm{mg} / \mathrm{L}$ & Usually coma and metabolic abnormalities \\
$>1000 \mathrm{mg} / \mathrm{L}$ & $\begin{array}{l}\text { Life-threatening multiorgan dysfunction - metabolic abnormalities, cerebral oedema, } \\
\text { bone marrow suppression }\end{array}$ \\
$>2000 \mathrm{mg} / \mathrm{L}$ & Death expected without urgent haemodialysis \\
\hline
\end{tabular}

Source: reference 4

\section{REFERENCES}

1. Martin E, Tozer TN, Sheiner LB, Riegelman S. The clinical pharmacokinetics of phenytoin. J Pharmacokinet Biopharm 1977;5:579-96. https://doi.org/10.1007/BF01059685

2. Hermida J, Tutor JC. A theoretical method for normalizing total serum valproic acid concentration in hypoalbuminemic patients. J Pharmacol Sci 2005;97:489-93. https://doi.org/ 10.1254/jphs.FPE04007X
3. Patsalos PN, Spencer EP, Berry DJ. Therapeutic drug monitoring of antiepileptic drugs in epilepsy: a 2018 update. Ther Drug Monit 2018;40:526-48. https://doi.org/10.1097/ FTD.0000000000000546

4. Murray L, Little M, Pascu O, Hogget K, editors. Toxicology handbook. 3rd ed. Chatswood: Elsevier Australia; 2015 